\title{
هִ The medial wall of the cavernous sinus. Part 1: Surgical anatomy, ligaments, and surgical technique for its mobilization and/or resection
}

\author{
Huy Q. Truong, MD, Stefan Lieber, MD, Edinson Najera, MD, Joao T. Alves-Belo, MD, \\ Paul A. Gardner, MD, and Juan C. Fernandez-Miranda, MD \\ Department of Neurological Surgery, University of Pittsburgh School of Medicine, Pittsburgh, PA
}

OBJECTIVE The medial wall of the cavernous sinus (CS) is often invaded by pituitary adenomas. Surgical mobilization and/or removal of the medial wall remains a challenge.

METHODS Endoscopic endonasal dissection was performed in 20 human cadaver heads. The configuration of the medial wall, its relationship to the internal carotid artery (ICA), and the ligamentous connections in between them were investigated in 40 CSs.

RESULTS The medial wall of the CS was confirmed to be an intact single layer of dura that is distinct from the capsule of the pituitary gland and the periosteal layer that forms the anterior wall of the CS. In $32.5 \%$ of hemispheres, the medial wall was indented by and/or well adhered to the cavernous ICA. The authors identified multiple ligamentous fibers that anchored the medial wall to other walls of the CS and/or to specific ICA segments. These parasellar ligaments were classified into 4 groups: 1) caroticoclinoid ligament, spanning from the medial wall and the middle clinoid toward the clinoid ICA segment and anterior clinoid process; 2) superior parasellar ligament, connecting the medial wall to the horizontal cavernous ICA and/or lateral wall of the CS; 3 ) inferior parasellar ligament, bridging the medial wall to the anterior wall of the CS or anterior surface of the short vertical segment of the cavernous ICA; and 4) posterior parasellar ligament, which anchors the medial wall to the short vertical segment of the cavernous ICA and/or the posterior carotid sulcus. The caroticoclinoid ligament and inferior parasellar ligament were present in most CSs (97.7\% and 95\%, respectively), while the superior and posterior parasellar ligaments were identified in approximately half of the CSs (57.5\% and $45 \%$, respectively). The caroticoclinoid ligament was the strongest and largest ligament, and it was typically assembled as a group of ligaments with a fan-like arrangement. The inferior parasellar ligament was the first to be encountered after opening the anterior wall of the CS during an interdural transcavernous approach.

CONCLUSIONS The authors introduce a classification of the parasellar ligaments and their role in anchoring the medial wall of the CS. These ligaments should be identified and transected to safely mobilize the medial wall away from the cavernous ICA during a transcavernous approach and for safe and complete resection of adenomas that selectively invade the medial wall.

https://thejns.org/doi/abs/10.3171/2018.3.JNS18596

KEYWORDS medial wall of cavernous sinus; parasellar ligament; endoscopic endonasal transcavernous; medial wall resection; anatomy; pituitary surgery; surgical technique

$\mathrm{T}$ HE structure of the cavernous sinus (CS) has been compared to the hull of a boat, as it is narrow at its anterior-most aspect (the transition from the CS into the superior orbital fissure) and wide posteriorly where it opens into the venous confluence formed by the basilar and petrosal sinuses. Rhoton described the CS as having 4 walls: lateral, medial, posterior, and superior (or roof). ${ }^{21}$ Yasuda et al. described the medial wall as consisting of 2 parts: a sellar part, which covers the pituitary gland, and a sphenoidal part, which faces the sphenoid sinus. ${ }^{24}$ In a previous publication, however, based on the dural architecture of the sellar and parasellar region, we proposed the identification of an anterior wall of the CS in place of the sphenoidal part and its differentiation from the medial wall of the CS, correspondent to Yasuda's sellar part. ${ }^{1}$

The medial wall of the CS forms the lateral boundary of the hypophyseal fossa and separates the pituitary gland from the cavernous segment of the internal carotid artery 
(ICA) and the venous channels. While there has been significant confusion regarding the anatomy and even the existence of the medial wall of the $\mathrm{CS},{ }^{13}$ several authors have demonstrated that the medial wall does exist as a distinct anatomical structure different than the pituitary capsule. $3,4,8,20,24,25$

Surgical removal and/or mobilization of the medial wall of the CS remains a surgical challenge. Complete surgical removal of the medial wall of the CS, as required for resection of some invasive pituitary adenomas, is key to achieve remission in cases of functional adenoma and reduce recurrence rates in cases of nonfunctional adenoma. The recent introduction of the endoscopic endonasal transcavernous posterior clinoidectomy technique ${ }^{11}$ has significantly improved, in our experience, surgical access for resection of lesions such as chordomas, chondrosarcomas, petroclival meningiomas, and retroclival epidermoid tumors. This technique requires mobilization of the medial wall of the CS to perform an interdural pituitary transposition and to provide the corridor to access the posterior clinoid, oculomotor triangle, and lateral recess of the interpeduncular cistern.

The aim of this study was to investigate the surgical anatomy of the medial wall of the CS and to describe an anatomy-based step-by-step surgical technique as required for safe and effective removal and/or mobilization of the medial wall of the CS. In part 2 of this study, ${ }^{7}$ we report the surgical outcomes in a large series of cases of invasive pituitary adenoma in which selective removal of the medial wall of the CS was performed using the surgical technique described here.

\section{Methods}

Twenty-five lightly embalmed human head specimens were used for this study. Twenty of them were dissected endonasally (medial-to-lateral dissection) with the aid of endoscopes; 12 of these specimens were injected with colored latex, and to facilitate intracavernous dissections the remaining 8 specimens were not injected. Five injected specimens were used for transcranial (lateral-to-medial) microsurgical dissection and for comparative purposes, but were not used for data collection. The configuration of the medial wall of the CS, its relation to the adjacent cavernous ICA, and the ligamentous connections between the wall, the ICA, the anterior clinoid process, and other walls of the CS were investigated. Surgical removal of the medial wall of the CS was illustrated with step-by-step anatomical dissections.

\section{Results}

\section{Medial Wall of the CS and the Pituitary Capsule}

The dura mater that covers the sellar floor and front is composed of 2 layers-meningeal or inner and periosteal or outer. At the junction between the sellar floor and the carotid sulcus, the 2 layers split and course away from each other: the outer layer continues laterally to form the anterior wall of the CS (or sphenoidal part, as it was called by Yasuda et al. ${ }^{24}$ ), while the inner layer remains attached to the pituitary gland to form the medial wall (or sellar part) of the CS (Fig. 1A and B). Consequently, both the anterior and medial walls of the CS are single layered and have distinct origins. Along the sellar floor and front, the 2 layers are separated by venous blood running along various intercavernous channels, often organized as inferior, posterior, and superior intercavernous sinuses. At the level of the posterior gland, the 2 layers are less readily dissected when compared to the anterior floor portion. Superiorly, the medial wall of the CS continues as the meningeal layer of the dural diaphragm and covers the upper aspect of the gland but leaves a portion of the gland uncovered at the diaphragmatic aperture (Fig. 1A).

In this study, the medial wall of the CS was distinct from and loosely connected to the pituitary capsule, which is a thin layer of connective tissue that tightly attaches to the glandular tissue (Fig. 1C and D). The loose adhesions between the pituitary capsule and the medial wall of the CS were thin irregular arachnoid-like bands of connective tissue that, despite having been previously described as "pituitary ligaments,"14 did not have a true ligamentous or dura-like structure. These loose adhesions were easily separated from the medial wall of the CS with blunt dissection. After dissecting the pituitary gland and capsule off the medial wall, we inspected its sellar or medial surface (Fig. 1D). In all cases, the medial wall was formed by a complete and well-defined dura-like membrane, which typically had a slightly convex shape due to the pituitary gland configuration. In a third (32.5\%) of cases, however, a portion of the medial wall of the CS was slightly concave as it was compressed from lateral to medial by the cavernous ICA, which was looping medially (Fig. 1D). This compression and adherence of the cavernous ICA to the medial wall of the CS did not affect the completeness and integrity of the medial wall.

\section{Medial Wall of the CS and Parasellar Ligaments}

Multiple ligamentous dura-like trabeculae were found connecting and anchoring the cavernous (lateral) surface of the medial wall of the CS to the anterior wall, the ICA, the lateral wall, the interclinoid ligament, and the anterior clinoid process, with much variation in shape, size, and trajectory. We proposed that these ligamentous trabeculae be named parasellar ligaments and we classified them into 4 categories based on their trajectory, as follows.

1. Caroticoclinoid ligament: This is the strongest and most constant ligament. It was present in $97.5 \%$ of cases. This ligament consists of a varying number of fibrous bundles from the proximal ring, middle clinoid, and the upper portion of medial wall that converge laterally into a strong band of connective tissue that loops behind the anterior genu of the ICA to eventually merge with the interclinoid ligament posteriorly and insert to the anterior clinoid process at its inferior surface (Figs. 2 and 3). This ligament suspends and anchors the medial wall of the CS superiorly (Fig. 3A and B). The caroticoclinoid ligament is typically tightly adhered to the wall of the ICA genu, and it forms the medial aspect of the proximal dural ring (Fig. 3C). Therefore, it marks the transition from the cavernous to the paraclinoid segment of the ICA, and it separates medially 

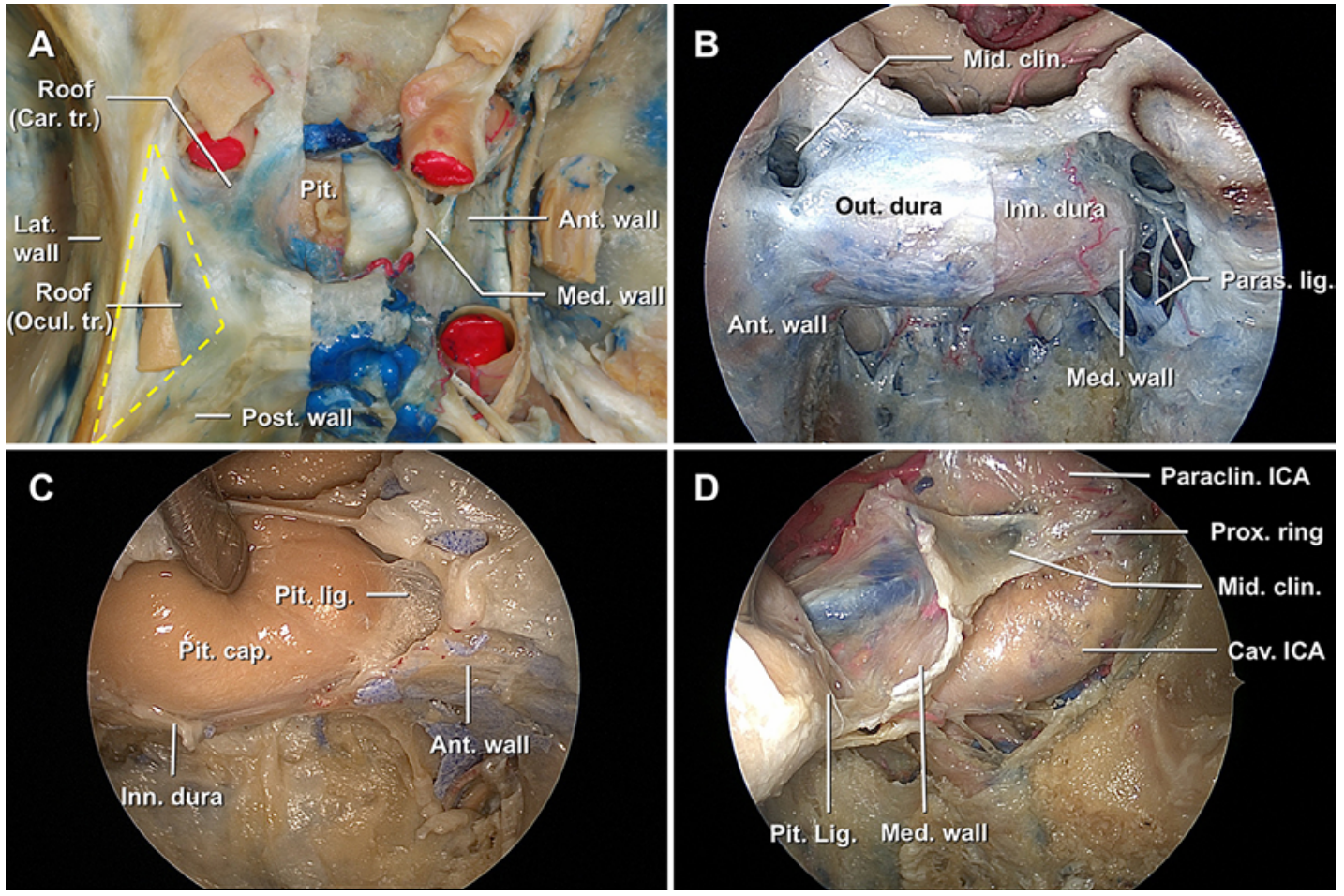

FIG. 1. Medial wall of the CS. A: Superior view of a half-dissected sellar and parasellar region showing the relative position and relationship between the walls and roof of the CS. Yellow dashed lines mark the oculomotor triangle or posterior roof of the CS. B: Endoscopic anteroinferior view of a half-dissected sellar floor exhibiting the double-layered nature of the sellar floor, the transition of the outer dura (periosteal) layer from the sellar floor to the anterior wall of the CS, and the coursing of the inner dural layer toward the diaphragm and forming the medial wall of the CS. The parasellar ligaments can be seen attached to the medial wall. C: Endoscopic view of the sellar fossa after removal of the sellar floor, showing the pituitary gland wrapped inside the thin and transparent pituitary capsule and its loose connections, or pituitary ligaments, to the medial wall of the CS. D: Endoscopic view of the sellar fossa with mobilization of the pituitary gland to show the medial or sellar surface of the medial wall of the CS. A slight indentation of the wall by the ICA can be seen. The impression of the middle clinoid (removed), together with the proximal ring, serves as a good landmark for the caroticoclinoid ligament (present in Figs. 2 and 3 ) and the transition between cavernous and paraclinoid segments of the ICA. Ant. wall = anterior wall; Car. tr. = carotid triangle; Cav. ICA = cavernous ICA; Inn. dura = inner dura; Lat. wall = lateral wall; Med. wall = medial wall; Mid. clin. = middle clinoid; Ocul. tr. = Oculomotor triangle; Out. dura = outer dura; Paraclin. ICA = paraclinoid ICA; Paras. lig. = parasellar ligaments; Pit. = pituitary gland; Pit. cap. = pituitary capsule; Pit. lig. $=$ pituitary ligament; Post. wall = posterior wall; Prox. ring = proximal ring. Copyright Juan C. Fernandez-Miranda. Published with permission. Figure is available in color online only.

the CS (below) from the clinoid space (above). The caroticoclinoid ligament may appear as an intact broad ligamentous band or, most commonly, as a fenestrated one in form of several ligamentous bands (Fig. 2 and Fig. 3C), allowing, in the latter case, venous communication between the cavernous and clinoid spaces. The merged interclinoid and caroticoclinoid ligaments continue laterally as the carotid-oculomotor membrane, which completes the proximal dural ring laterally (Fig. $3 \mathrm{D})$. The interclinoid ligament is located at the same craniocaudal level and immediately posterior to the caroticoclinoid ligament. Both ligaments are intimately related as they run laterally toward the anterior clinoid process, but they diverge medially as the interclinoid ligament attaches to the posterior clinoid process and the caroticoclinoid ligament attaches to the middle clinoid process (Figs. 2 and 3).

2. Superior parasellar ligament: This ligament originates from the superior and posterior aspects of the medial wall of the CS, usually at the same level of the caroti- coclinoid ligament but with a different trajectory as it bridges the medial wall with the horizontal portion of the cavernous ICA and/or the lateral wall of the CS. The ligament is usually thin and has a fan-like form (Fig. 4A and B). The superior parasellar ligament was found in only $57.5 \%$ of specimens, and when present, it separated the superior and inferior venous compartments of the CS.

3. Inferior parasellar ligament: This ligament connects the medial wall to the anterior wall of the CS and/or the short vertical portion of the cavernous ICA. It typically arises from the anterior and inferior aspects of the medial wall, but it can also originate from the caroticoclinoid (Fig. 4C-E). The inferior parasellar ligament can be single or multiple, as we found up to 3 inferior parasellar ligaments in a single CS. It was present in $95 \%$ of specimens, and it is typically the first ligament to be encountered during a transcavernous approach after opening the anterior wall of the CS.

4. Posterior parasellar ligament: This is the least prevalent 
ligament, as it was identified in only $45 \%$ of specimens. The posterior parasellar ligament anchored the posterior portion of the medial wall of the CS to the posterior aspect of the short vertical portion of the cavernous ICA and/or to the periosteal layer of the posterior wall of the carotid sulcus (Fig. 4F). The posterior parasellar ligament is usually the smallest ligament as well. Importantly, it is closely related to the inferior hypophyseal artery in $83 \%$ of cases, when present.

In addition to studying the parasellar ligaments individually, we also considered them all together and investigated the anchoring pattern of the medial wall of the CS. We found 3 differential patterns of arrangement of the medial wall of the CS and its ligaments: 1) independent anchoring (62\%), in which the parasellar ligaments arise independently from different aspects of the medial wall (superior, inferior, posterior), as detailed above; 2) caroticoclinoid ligament anchoring (32\%), where the fibers of the inferior parasellar ligament attach to the medial wall via the caroticoclinoid ligament; and 3) common anchoring $(6 \%)$, in which all ligaments are joined to form a strong common attachment to the medial wall.

In cases of mobilization of the medial wall for an interdural pituitary transposition, the transection of the caroticoclinoid ligament is critical to maximizing the mobilization of the medial wall and pituitary gland, to increasing the exposure of prominent posterior clinoids, and to providing access to the posterior wall of the CS, oculomotor triangle dura, and lateral interpeduncular cistern, when invaded by tumor.

The intraoperative appearance and the parasellar ligament transection technique are illustrated through several short video clips extracted from recorded surgeries (Video 1).

VIDEO 1. Intraoperative recognition and transection of parasellar ligaments. Copyright Juan C. Fernandez-Miranda. Published with permission. Click here to view.

\section{Endoscopic Endonasal Selective Mobilization and Removal of the Medial Wall of the CS: Technical Nuances}

From the findings on the structure of the medial wall of the CS, its relationship to the ICA, and the ligamentous connections inside the CS, we proposed an anatomically based step-by-step technique for safe mobilization and resection of the medial wall of the CS (Fig. 5).

\section{Step 1: Sellar and Parasellar Exposure}

The standard endonasal endoscopic surgical approach to the sphenoid sinus is performed as described elsewhere. ${ }^{25}$ Once the sphenoid sinus opening has been maximized, extensive sellar and parasellar exposure, including the anterior wall of the CS and paraclinoid segment of the ICA, is completed. The middle clinoid, when present, serves as a reference for the location of the roof of the CS and the transition between the cavernous and paraclinoid ICA segments (Fig. 1D and Fig. 3A and C). Removal of the middle clinoid is required, except when a caroticoclinoid osseous ring is identified; in this case, reduction of the middle clinoid is sufficient and safer. The surgical steps to accomplish a middle clinoidectomy have been described

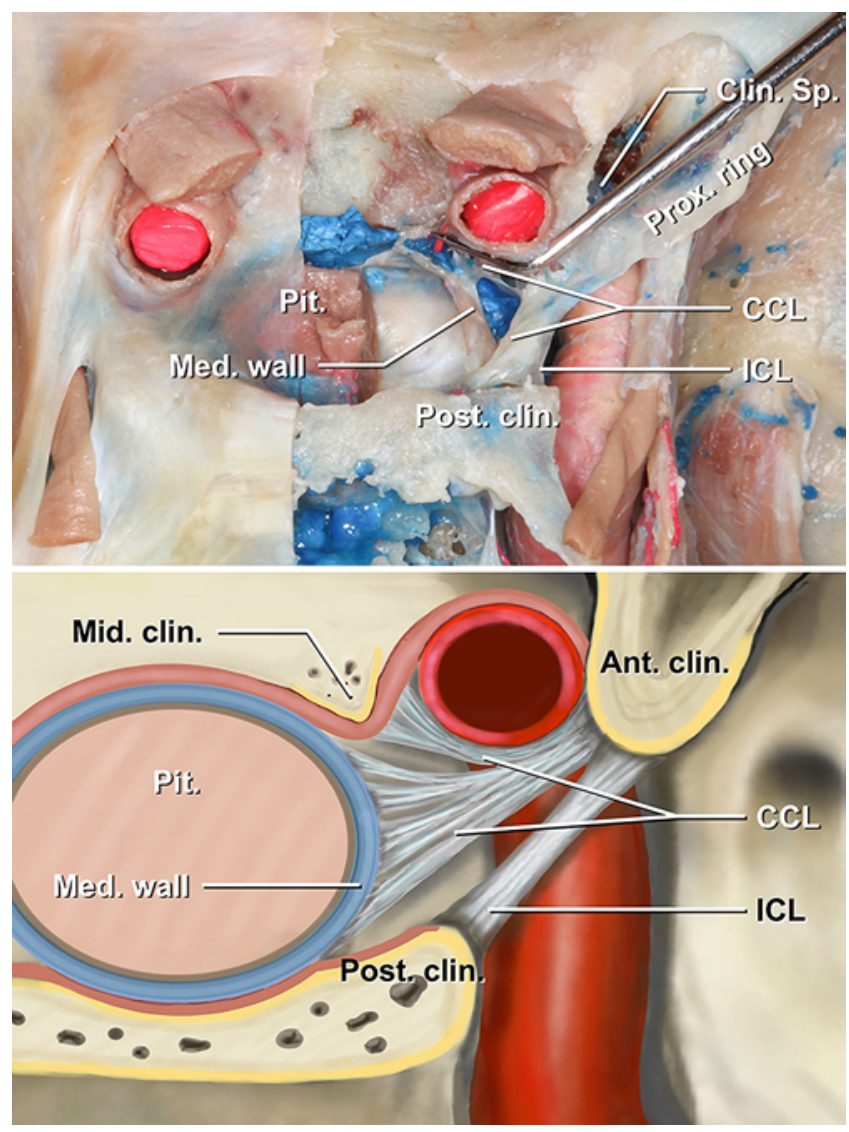

FIG. 2. Microscopic view of the caroticoclinoid ligament. Upper: Superior view of a half-dissected sellar and parasellar region showing a caroticoclinoid ligament that originates from the medial wall of the CS and attaches to the anterior clinoid process and continues with the proximal ring. The ligament has a large fenestration, which allows venous communication between the CS below and the clinoid space above. The dissection also shows the tight relationship between the caroticoclinoid ligament and the interclinoid ligament as they usually blend laterally before they both attach to the inferior surface of the anterior clinoid process. Lower: Schematic illustration that simplifies the anatomy of the upper panel and shows the 2 components of the caroticoclinoid ligament from the middle clinoid and medial wall of the CS and the relationship to the ICL. Ant. clin. = anterior clinoid; $\mathrm{CCL}=$ caroticoclinoid ligament; Clin. $\mathrm{Sp}$. = clinoid space; $\mathrm{ICL}=$ interclinoid ligament; Med. wall = medial wall; Mid. clin. = middle clinoid; Pit. = pituitary gland; Post. clin. = posterior clinoid; Prox. ring = proximal ring. Copyright Juan C. Fernandez-Miranda. Published with permission. Figure is available in color online only.

elsewhere. ${ }^{12}$ The exposure of the sellar dura continues superiorly up to the distal dural ring and tuberculum sellae, which typically does not have to be removed unless suprasellar access is required. Inferiorly, it is important to drill out the sellar floor all the way back to the dorsum sellae. Then the bone that covers the most inferior aspect of the anterior wall of the CS is carefully removed; this is an essential step that exposes the critical area where the paraclival segment of the ICA enters the CS to become the short vertical segment of the cavernous ICA (Fig. 5A).

\section{Step 2: Sellar Tumor Removal and Medial Wall Inspection}

After this extensive bony work, the sellar dura is opened in a cruciate fashion. The superolateral limit of the 

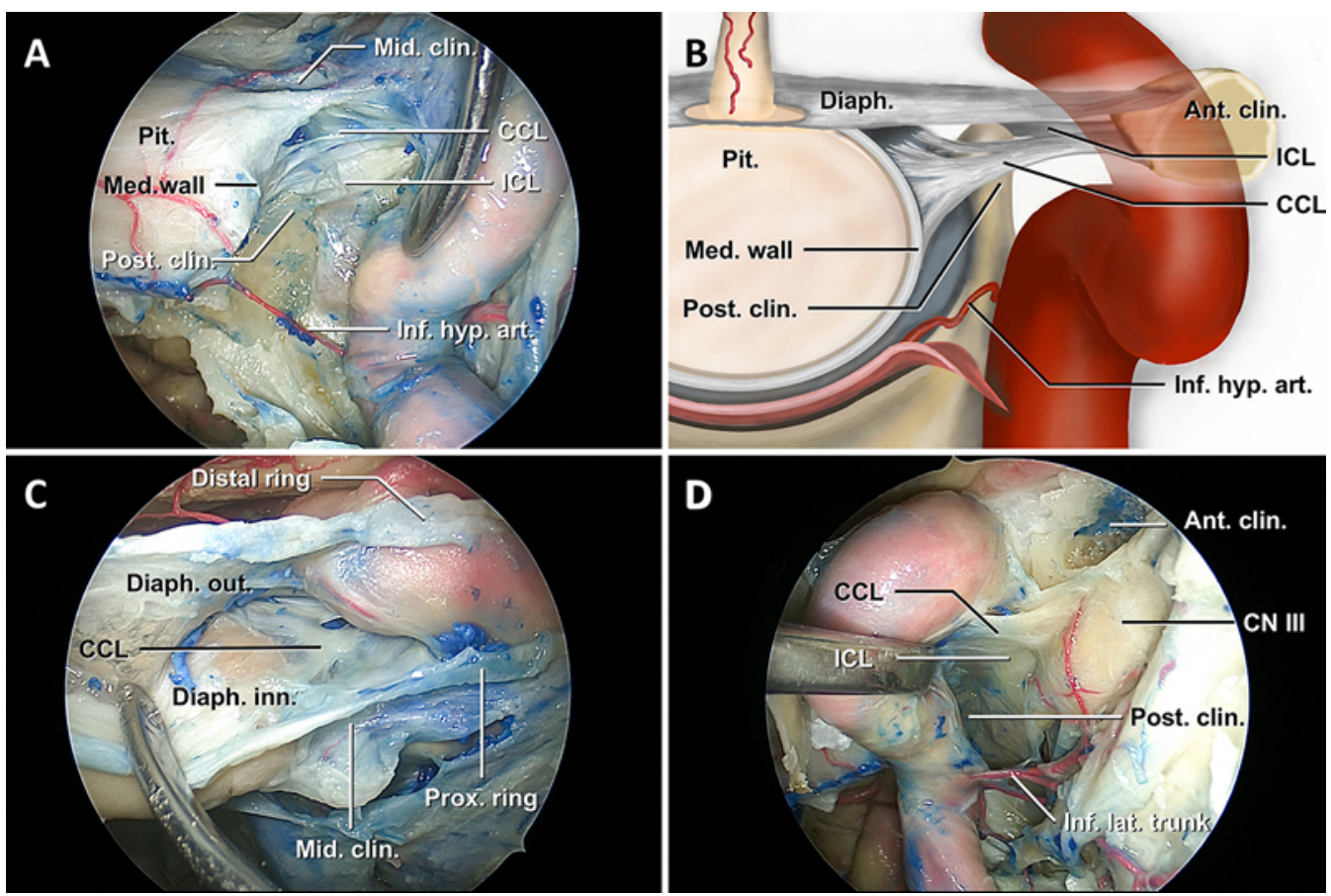

FIG. 3. Endoscopic view of the caroticoclinoid ligament. A: Image obtained after the anterior wall and periosteal sellar floor have been removed and the ICA has been mobilized laterally to provide an anteroinferior view of the caroticoclinoid ligament. The ligament shows continuity with the dura of the medial wall. The posterior clinoid and interclinoid ligament can be clearly seen. The inferior hypophyseal artery originating from the posterior genu of the ICA is seen crossing the CS just anterior to the posterior clinoid and reaching the pituitary gland at the interlobar fissure. B: Schematic drawing illustrating the anatomy showed in panel A. C: Anterior endoscopic view of the upper sellar and paraclinoid region. The clinoid space was opened from lateral to medial, and the dissection was continued medially to separate the 2 layers of the diaphragma sellae-the inner layer and the outer layer, which are in continuity with the medial wall and roof of the CS, respectively. This view shows the construction of the caroticoclinoid ligament from bundles of fibers from the proximal ring via the middle clinoid and from the medial wall of the CS. A fenestration and venous connection between the clinoid space and CS can be seen. D: Endoscopic view of the lateral wall of the CS obtained by mobilizing the anterior genu of the ICA medially. This view shows the attachment of the caroticoclinoid ligament and interclinoid ligament to the inferior surface of the anterior clinoid process and their continuity with the proximal ring and oculomotor membrane. Med. wall = medial wall; Mid. clin. = middle clinoid; Pit. = pituitary gland; Post. clin. = posterior clinoid; Ant. clin. = anterior clinoid; $C C L=$ caroticoclinoid ligament; CN III = third cranial nerve; Diaph. = diaphragma sellae; Diaph. inn. = diaphragm inner layer; Diaph. out. = diaphragm outer layer; $\mathrm{ICL}=$ interclinoid ligament; Inf. lat. trunk = inferolateral trunk; Inf. hyp. art. = inferior hypophyseal artery; Prox. ring = proximal ring. Copyright Juan C. Fernandez-Miranda. Published with permission. Figure is available in color online only.

dural cut is marked by the location of the middle clinoid, which corresponds to the location of the roof of the CS medially. The inferolateral dural cut is stopped just before the divergence of the dural layers at the floor of the sella. After the dural opening, the resection of the adenoma is performed in an extracapsular fashion whenever feasible. In cases of true selective invasion of the medial wall, tumor components remain attached to the medial wall and cannot be separated. At the same time, the medial wall is mostly intact, preventing the tumor from spreading into the inside of the CS. In cases of mobilization of the medial wall for an interdural pituitary transposition, as required for chordomas and other extrasellar lesions, this step 2 is not required, and the sellar dura is not opened (Fig. 5B).

\section{Step 3: Opening the Anterior Wall of the CS}

The next step consists of the identification of the point of separation between the periosteal and meningeal dural layers at the floor and front of the sella to gently start mobilizing the medial wall. This is facilitated by opening the anterior wall of the CS directly. The safest entry zone into the CS is usually at the lowest aspect of the anterior wall, where there is frequently a large venous space between the cavernous ICA and the medial wall of the CS due to the inferior petrosal sinus opening into the CS. An intraoperative Doppler probe should be used to identify the trajectory of the carotid artery before opening the anterior wall of the CS. Prominent venous bleeding is controlled with intracavernous injection of Surgifoam (Ethicon, Inc.) and gentle packing with cottonoids. These hemostasis maneuvers are repeated as many times as needed during the approach. Then the opening can be widened by using a blunt-tipped right-angle knife (McElveen knife) in a lateral direction below the anterior ICA genu and in a superior direction toward the middle clinoid and medial to the anterior ICA genu (Fig. 5C).

Step 4: Transcavernous Approach to Release the Anchoring of the Medial Wall of the CS

At this point, the cavernous ICA and its branches (inferior hypophyseal artery) should be recognized. An intraoperative Doppler probe is again used to identify the trajec- 

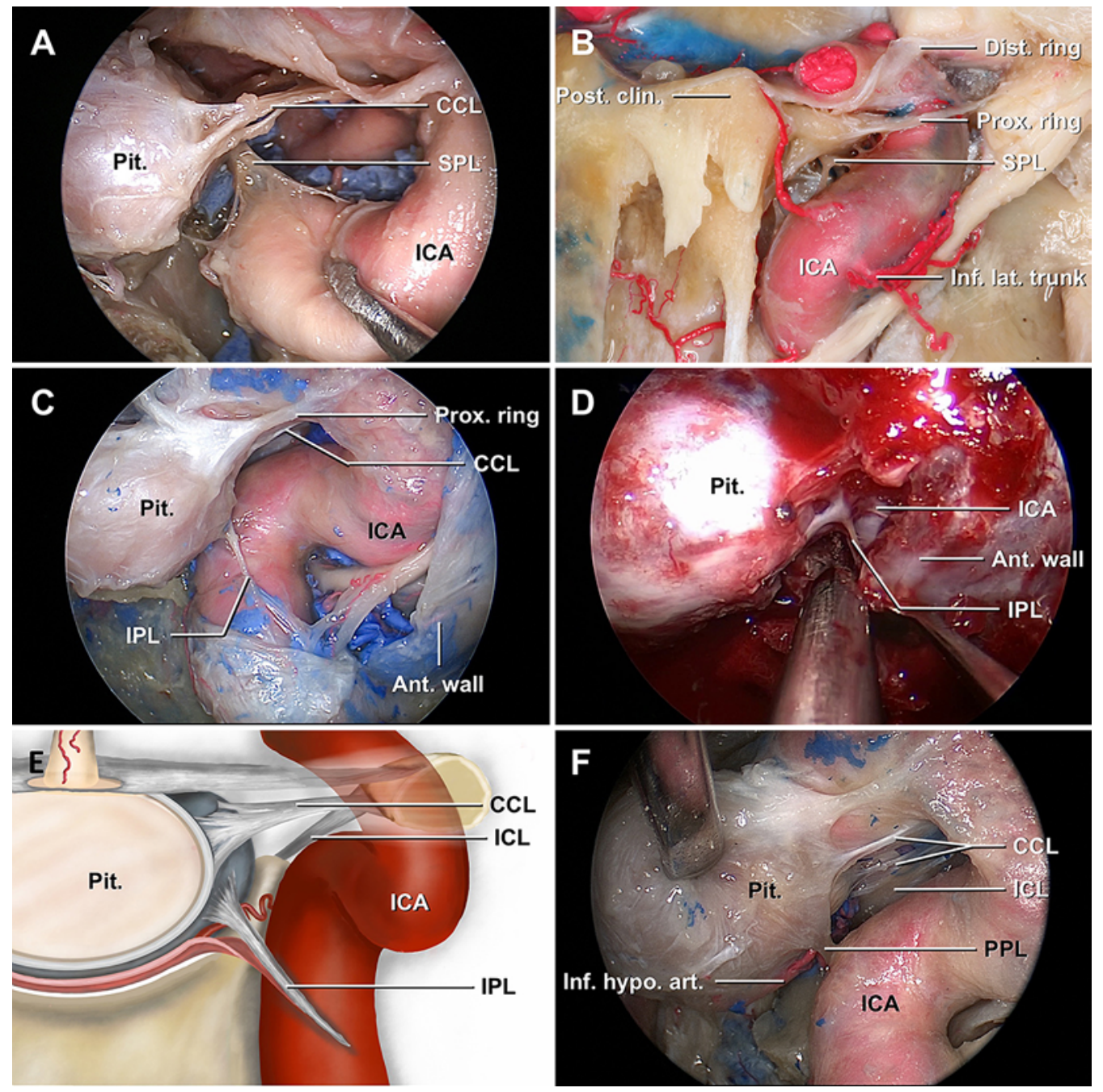

FIG. 4. Parasellar ligaments. A: Endoscopic view of the CS showing the direction and attachment of the superior parasellar ligament to the horizontal cavernous ICA. B: Superolateral view of the CS, revealing the attachment of the superior parasellar ligament on the upper wall of the horizontal cavernous ICA. Interestingly, in this specimen, there is ossification of both the interclinoid and caroticoclinoid ligaments. C: Endoscopic view showing a slim inferior parasellar ligament bridging the medial wall to the anterior wall of the CS. D: Intraoperative photograph of the rare common anchoring pattern, in which the inferior parasellar ligament and caroticoclinoid ligament (partly hidden) blend and form an X-shaped common ligament. E: Schematic illustration that simplifies the anatomy of the caroticoclinoid ligament, interclinoid ligament, and inferior parasellar ligaments. F: Subsequent dissection of the specimen in panel $\mathrm{C}$ shows the posterior parasellar ligament, which is quite thick in this case. The inferior hypophyseal artery can be seen coursing very close to the posterior parasellar ligament. The interclinoid and caroticoclinoid ligaments can also be identified. Ant. wall = anterior wall; $C C L=$ caroticoclinoid ligament; Dist. ring = distal ring; Inf. lat. trunk = inferolateral trunk; Inf. hypo. art. = inferior hypophyseal artery; IPL = inferior parasellar ligament; Pit. = pituitary gland; Post. clin. = posterior clinoid; Prox. ring $=$ proximal ring; $\mathrm{PPL}=$ posterior parasellar ligament; $\mathrm{SPL}=$ superior parasellar ligament. Copyright Juan C. Fernandez-Miranda. Published with permission. Figure is available in color online only.

tory of the carotid artery within the CS. The ligaments that anchor the medial wall to the ICA and to the other walls of the CS are now recognized in thickness and configuration. The anchoring pattern of the medial wall of the CS should now be identified as described above, as this determines the required transection sequence. Most frequently, the first ligament to be encountered is the inferior parasellar ligament, which is attached to the short vertical portion of the carotid artery. The next ligament, when present, is the superior parasellar ligament, which is attached to the horizontal portion of the carotid artery. Following transection of these ligaments, dissection of the medial wall from the ICA is started. Then, dissection can, and should, be done toward the medial wall to avoid manipulation of the ICA and minimize potential complication. The inferior hypophyseal artery should then be identified as it typically runs between the inferior and posterior parasellar ligaments, and it can be mistakenly identified as part of them. The inferior hypophyseal artery is selectively coagulated and transected to avoid the risk of avulsion of the artery from the wall of the ICA. This step is key in allowing further mobilization of the medial wall away from the ICA and progressing in a posterior direction, all the way to the posterior clinoid process and dorsum sellae, and in a superior direction all 

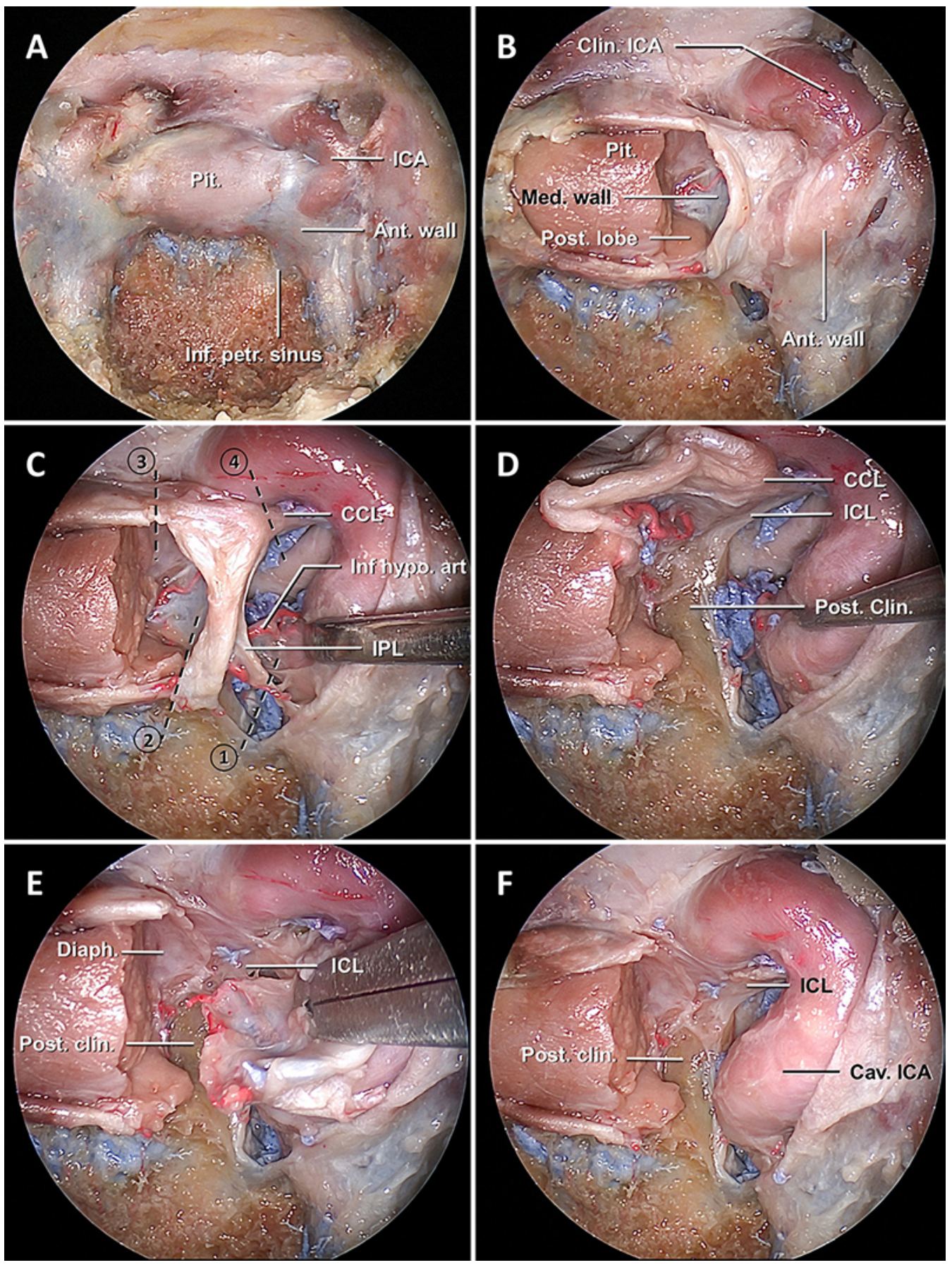

FIG. 5. Step-by-step medial wall resection. A: The exposure should include the sella, clinoid ICA, and anterior wall of the CS and can extend inferiorly to reveal the opening of inferior petrosal sinus into the CS. B: After removal of tumor, the medial surface of the medial wall of the CS should be inspected for tumor invasion. C: The transcavernous approach directly opens the anterior wall to expose and transect the ligaments and release the medial wall for resection. The numbered dashed lines show the location and order of the sequential cuts needed for complete resection of the medial wall. D: After the first cut through the inferior parasellar ligament, the medial wall can then be mobilized medially away from the ICA. Then, the second cut is performed through the sellar floor just lateral to posterior lobe and toward the dorsum sellae and posterior clinoid. E: The third cut separates the medial wall from the inner diaphragm (sellar roof) medially. F: After transecting the caroticoclinoid ligament, the medial wall is completely resected, and the interclinoid ligament and posterior clinoid are exposed. Ant. wall = anterior wall; Cav. ICA = cavernous ICA; $\mathrm{CCL}$ = caroticoclinoid ligament; Clin. ICA = clinoid ICA; Diaph = diaphragm sellae; ICL = interclinoid ligament; Inf hypo. art. = inferior hypophyseal artery; Inf. petr. sinus, inferior petrosal sinus; IPL = inferior parasellar ligament; Med. wall = medial wall; Pit. = pituitary gland; Post. clin. = posterior clinoid; Post. lobe = posterior lobe of pituitary gland. Copyright Juan C. Fernandez-Miranda. Published with permission. Figure is available in color online only. 
the way up to the diaphragm and caroticoclinoid ligament, which attaches to the paraclinoid ICA (Fig. 5C).

\section{Step 5: Dural Cuts for Removal of the Medial Wall of the CS}

The last step is to complete the dural cuts: a) medial and inferior dural cut, which separates the medial wall from the dural floor that covers the posterior gland and continues over the dorsum sellae toward the posterior clinoid (Fig. 5D); b) superior dural cut, which disconnects the medial wall from the sellar diaphragm (Fig. 5E); c) lateral cut, which disconnects the medial wall from the paraclinoid ICA and proximal dural ring - this last cut can be done across the caroticoclinoid ligament and therefore exposing the interclinoid ligament (posterior to the caroticoclinoid ligament) or just below the caroticoclinoid ligament to finally remove the medial wall (Fig. 5F). The transection of the caroticoclinoid ligament starts just medial to the anterior genu/paraclinoid ICA and then continues in an anterior-to-posterior direction to sharply interrupt all the fibrous bands that form the caroticoclinoid ligament, until the interclinoid ligament and dura of the oculomotomor triangle are identified posteriorly to confirm the complete transection of the caroticoclinoid ligament.

In cases of minimal invasion, the medial wall can be removed by transecting the dura just below the caroticoclinoid ligament; this decreases the difficulty and risk of the procedure.

An illustrative case of Cushing's disease in which we did remove the medial wall of the CS during the tumor resection is presented in Video 2.

VIDEO 2. Resection of medial wall of the CS during tumor removal for treatment of Cushing's disease. Copyright Juan C. Fernandez-

Miranda. Published with permission. Click here to view.

\section{Discussion}

The medial wall of the CS, due to its critical importance in the evaluation and treatment of pituitary adenomas, has been a long-pursued subject studied with many methodologies, including microsurgical anatomy, $2,3,8,15,20,24$ radiology, ${ }_{10}$ histology, $4,9,25$ immunohistochemistry, $4,17,20,22$ and embryology. ${ }^{5,6,16,18,23}$ While there is still controversy about the origin and nature of the medial wall of the CS, it is clearly not a simple invagination of diaphragm dura by the descending neurohypophysis. The thickness of the wall and number of layers vary between studies, but most recent studies have shown that the wall is an intact layer with no perforation, $3,4,8,20,24,25$ as our findings confirm.

The ligamentous trabeculae-or parasellar ligamentsof the CS have been noticed by several authors. ${ }^{2,3,8,19,25}$ Most authors, however, limited their findings to merely noting the existence of these structures and suggesting their role in anchoring the pituitary gland, without discussing their clinical significance. These previous studies have predominantly employed lateral transcavernous microsurgical dissection as their study method, which requires removal of the lateral wall, anterior clinoid process, and cavernous ICA to expose the CS and its medial wall. This lateral-tomedial approach to the CS is likely to disrupt the ligamentous trabeculae of the CS.

Two previous authors did elaborate on the variation and trajectory of the parasellar ligaments., ${ }^{3,19}$ The earliest ef- fort in classifying the ligaments was by Lang ${ }^{19}$ however, it is difficult to correlate his findings with ours due to the small number of specimens and the paucity of illustrations in his study. A later description by Buric et al. ${ }^{3}$ was more consistent with our findings, but the authors' interpretation of the anchoring of the medial wall to the middle and posterior clinoid processes differs from ours. Importantly, in their study they did not elaborate on the surgical implication of their findings to develop an anatomically based technique for mobilization and/or resection of the medial wall of the CS.

From our observation of the parasellar ligaments, we prefer the theory of mesenchymal origin proposed by Kehrli et al. ${ }^{16}$ The authors demonstrated with fetal histological sections at different stages that the fetal pituitary gland was surrounded by a mass of mesenchyme, which later developed venous channels that became larger and larger and condensed around the gland. The final result was a capsule wrapped around the pituitary gland and attached to the periosteal layer of the sphenoid bone along the sellar floor, forming the medial wall of the CS bilaterally and forming the diaphragm superiorly through its attachment to the meningeal dura.. The parasellar ligaments are remnants of the mesenchyme left after the expansion of the venous spaces. This theory explains well the variation of the ligaments and the different patterns of medial wall anchoring described here. In fact, the presence of parasellar ligaments running along and attaching to the medial wall of the CS may cause the variety in thickness of the medial wall reported in previous studies ${ }^{20,25}$ or the false assumption of double layering in a previous histological study..$^{22}$

Understanding the dural layering and walls of the CS, as well as the parasellar ligaments and anchoring patterns of the medial wall, has been key to developing a step-bystep technique for mobilizing and removing the medial wall of the CS. By opening the anterior wall directly, we can achieve better control of the venous bleeding from the CS. This also allows us to correctly identify the ligaments that anchor the medial wall to the cavernous ICA, selectively coagulate the inferior hypophyseal artery, and properly transect the ligaments and the artery to gradually detach and mobilize the medial wall away from the cavernous ICA. This mobilization of the medial wall is the basis for the interdural pituitary transposition technique that has proven very effective for the removal of posterior clinoids involved with tumor, such as chordomas and chondrosarcomas, and for expanding the surgical corridor to access the oculomotor triangle and lateral recess of the interpeduncular cistern, as required for selective chondroid lesions, petroclival meningiomas, and retroclival epidermoid tumors.

In the case of pituitary adenomas with potential invasion of the medial wall of the CS, the commonly used medial-to-lateral technique to access the medial wall may carry a higher risk of injuring the ICA, as the artery is approached blindly through the medial wall, especially when considering the possibility of intimate adherence between the medial wall and the cavernous ICA, ${ }^{3,24}$ as found in a third of our specimens. We believe that direct visualization of the medial wall-ICA dissection plane will potentially offer a lower risk of ICA injury and will facilitate a complete removal of the medial wall using the landmarks and technique described here. The results of the proposed 
technique for invasive adenomas will be reported in part 2 of this study.

\section{Conclusions}

In this paper, we introduce a classification of the parasellar ligaments and their role in anchoring the medial wall of the CS. These ligaments should be identified and transected to safely mobilize the medial wall away from the cavernous ICA during a transcavernous approach and for safe and complete resection of adenomas that invade the medial wall of the CS.

\section{References}

1. Barges-Coll J, Fernandez-Miranda JC, Prevedello DM, Gardner P, Morera V, Madhok R, et al: Avoiding injury to the abducens nerve during expanded endonasal endoscopic surgery: anatomic and clinical case studies. Neurosurgery 67:144-154, 2010

2. Bergland RM, Ray BS, Torack RM: Anatomical variations in the pituitary gland and adjacent structures in 225 human autopsy cases. J Neurosurg 28:93-99, 1968

3. Buric J, Alexandre A, Corò L, Azuelos A: Medial wall of the cavernous sinus. Riv Neuroradiol 15:197-203, 2002

4. Ceylan S, Anik I, Koc K, Kokturk S, Ceylan S, Cine N, et al: Microsurgical anatomy of membranous layers of the pituitary gland and the expression of extracellular matrix collagenous proteins. Acta Neurochir (Wien) 153:2435-2443, 2011

5. Chi JG, Lee MH: Anatomical observations of the development of the pituitary capsule. J Neurosurg 52:667-670, 1980

6. Ciric I: On the origin and nature of the pituitary gland capsule. J Neurosurg 46:596-600, 1977

7. Cohen-Cohen S, Gardner PA, Alves-Belo JT, Truong HQ, Snyderman CH, Wang EW, et al: The medial wall of the cavernous sinus. Part 2: Selective medial wall resection in 50 pituitary adenoma patients. J Neurosurg [epub ahead of print September 7, 2018. DOI: 10.3171/2018.5.JNS18595]

8. Destrieux C, Kakou MK, Velut S, Lefrancq T, Jan M: Microanatomy of the hypophyseal fossa boundaries. J Neurosurg 88:743-752, 1998

9. Diao Y, Liang L, Yu C, Zhang M: Is there an identifiable intact medial wall of the cavernous sinus? Macro- and microscopic anatomical study using sheet plastination. Neurosurgery 73 (1 Suppl Operative):ons106-ons110, 2013

10. Dietemann JL, Kehrli P, Maillot C, Diniz R, Reis M Jr, Neugroschl C, et al: Is there a dural wall between the cavernous sinus and the pituitary fossa? Anatomical and MRI findings. Neuroradiology 40:627-630, 1998

11. Fernandez-Miranda JC, Gardner PA, Rastelli MM Jr, PerisCelda M, Koutourousiou M, Peace D, et al: Endoscopic endonasal transcavernous posterior clinoidectomy with interdural pituitary transposition. J Neurosurg 121:91-99, 2014

12. Fernandez-Miranda JC, Tormenti M, Latorre F, Gardner P, Snyderman C: Endoscopic endonasal middle clinoidectomy: anatomic, radiological, and technical note. Neurosurgery 71 (2 Suppl Operative):ons233-ons239, 2012

13. Gonçalves MB, de Oliveira JG, Williams HA, Alvarenga RMP, Landeiro JA: Cavernous sinus medial wall: dural or fibrous layer? Systematic review of the literature. Neurosurg Rev 35:147-154, 2012

14. Kassam AB, Prevedello DM, Thomas A, Gardner P, Mintz A, Snyderman C, et al: Endoscopic endonasal pituitary transposition for a transdorsum sellae approach to the interpeduncular cistern. Neurosurgery 62 (3 Suppl 1):57-74, 2008

15. Kawase T, van Loveren H, Keller JT, Tew JM: Meningeal architecture of the cavernous sinus: clinical and surgical implications. Neurosurgery 39:527-536, 1996
16. Kehrli P, Ali M, Reis M Jr, Maillot C, Dietemann JL, Dujovny M, et al: Anatomy and embryology of the lateral sellar compartment (cavernous sinus) medial wall. Neurol Res 20:585-592, 1998

17. Knappe UJ, Fink T, Fisseler-Eckhoff A, Schoenmayr R: Expression of extracellular matrix-proteins in perisellar connective tissue and dura mater. Acta Neurochir (Wien) 152:345-353, 2010

18. Kural C, Simsek GG, Guresci S, Arslan E, Kilic C, Tehli O, et al: Histological structure of the medial and lateral walls of cavernous sinus in human fetuses. Childs Nerv Syst 31:699703,2015

19. Lang J: Hypophyseal ligaments. Acta Neurochir (Wien) 130:144-146, 1994

20. Peker S, Kurtkaya-Yapicier O, Kiliç T, Pamir MN: Microsurgical anatomy of the lateral walls of the pituitary fossa. Acta Neurochir (Wien) 147:641-649, 2005

21. Rhoton ALJ Jr: The cavernous sinus, the cavernous venous plexus, and the carotid collar. Neurosurgery 51 (4 Suppl):S375-S410, 2002

22. Songtao Q, Yuntao L, Jun P, Chuanping H, Xiaofeng S: Membranous layers of the pituitary gland: histological anatomic study and related clinical issues. Neurosurgery 64 (3 Suppl):ons1-ons10, 2009

23. Tobenas-Dujardin AC, Duparc F, Laquerriere A, Muller JM, Freger P: Embryology of the walls of the lateral sellar compartment: apropos of a continuous series of 39 embryos and fetuses representing the first six months of intra-uterine life. Surg Radiol Anat 25:252-258, 2003

24. Yasuda A, Campero A, Martins C, Rhoton AL Jr, Ribas GC: The medial wall of the cavernous sinus: microsurgical anatomy. Neurosurgery 55:179-190, 2004

25. Yilmazlar S, Kocaeli H, Aydiner F, Korfali E: Medial portion of the cavernous sinus: quantitative analysis of the medial wall. Clin Anat 18:416-422, 2005

\section{Disclosures}

The authors report no conflict of interest concerning the materials or methods used in this study or the findings specified in this paper.

\section{Author Contributions}

Conception and design: Fernandez-Miranda, Truong. Acquisition of data: Truong, Lieber, Najera, Alves-Belo. Analysis and interpretation of data: Truong. Drafting the article: Truong. Critically revising the article: Fernandez-Miranda, Gardner. Reviewed submitted version of manuscript: Fernandez-Miranda, Gardner. Approved the final version of the manuscript on behalf of all authors: Fernandez-Miranda. Study supervision: FernandezMiranda.

\section{Supplemental Information \\ Videos}

Video 1. https://vimeo.com/269003927.

Video 2. https://vimeo.com/269004051.

\section{Companion Papers}

Cohen-Cohen S, Gardner PA, Alves-Belo JT, Truong HQ, Snyderman $\mathrm{CH}$, Wang EW, et al: The medial wall of the cavernous sinus. Part 2: Selective medial wall resection in 50 pituitary adenoma patients. DOI: 10.3171/2018.5.JNS18595.

\section{Correspondence}

Juan C. Fernandez-Miranda: Department of Neurological Surgery, Stanford University, Stanford, CA. drjfm@stanford.edu. 